\title{
Feature Based Hole Filling Algorithm on Triangular Mesh
}

\author{
Bin $\mathrm{Xu}^{1}$, Zhongke $\mathrm{Li}^{2}$, and Ying $\operatorname{Tan}^{3}$ \\ ${ }^{1}$ Teaching and Research Section of Computer, The Second Artillery Engineering University, \\ Xi`an, 710025, China \\ xubinliuxia@sina.com
}

\begin{abstract}
Aiming at the problem of triangular mesh hole repairing, a new geometric feature based hole filling algorithm was presented. The holes boundary were extracted and pretreated, and the advancing front mesh technique is used to cover the hole with newly created triangles; The Euclidencoordinate and lapacian of mesh points near the hole boundary were chosen as training sample for support vector machines and deduced lapacian of filling points; calculated coordinate of filling point throw possion equations based on deduced lapacian of filling points to file the hole precisely. Examples proved that this algorithm furbished geometric details to missing area of triangular mesh surface very good.
\end{abstract}

Keywords: Triangular mesh model, hole repairing, Laplacian, least-squares support vector machines, possion equation.

\section{Introduction}

Polygonal representations of 3D objects, and particular triangular meshes, have become prevalent in numerous application domains. As 3D optical scanners become widespread, triangle meshes can be easier created and widely applied in the fields of $\mathrm{CAD}$ and reverse engineering. Even with high-fidelity scanners, the data obtained is often incomplete. The existence of holes makes it difficult for many operations based on meshes, such as model rebuilding, rapid prototyping and finite element analysis. Therefore, certain repairs must be done before taking incomplete mesh models into actual applications, and hole-filling is the most important one among them. In many computer-aided engineering applications, detailed geometric features are very important, so how to make patched surface to match the missing geometry well is an core problem. Surround this center various mesh hole-filling approaches have been proposed in recent years.

Davis [1] apply a volumetric diffusion process to extend a signed distance function through this volumetric representation until its zero set bridges whatever holes may be present. Guo[2] employed space carving and iso-surface extraction to fill holes. Ju [3] constructed an inside or outside volume using an octree grid and re-constructed the surface by contouring. Joshua and Szymon [4] used a min-cut algorithm to split space into inside and outside portions, and patched the holes simultaneously in a globally 
sensitive manner. Holes with regular boundary over a relatively planar region can be easily patched via planar triangulation, which has been described in detail by a number of textbooks and papers [5, 6, 7]. However, filling a complex hole over an irregular region is much more difficult. To solve this problem, Carr [8] used radial basis function to construct an implicit surface to cover the hole. This method works well for convex surfaces and can handle irregular holes. But difficulties arise when the underlying surface is too complex to be described by a single-value function. Liepa [9] presented an umbrella operator to fair the triangulation over the hole to estimate the underlying geometry. However, the On3. performance of the triangulation method limits this method from being used widely. Jun [10] proposed a hole-filling method based on a piecewise scheme. His method divides a complex hole into several simple holes and all sub-holes are sequentially filled with planar triangulation; sub-division and refinement are then employed to smooth the new triangles. Chen [11] proposed a hole-filling method which can fill the hole and recover its sharp feature involved in the hole area. With this method, holes are filled using a radial basis function; a feature enhancement process based on Bayesian classification [12] and sharpness dependent filter [13] is then applied if there exists any sharp feature on the hole boundary. Some hole-filling algorithms for parametric surfaces have been presented $[14,15,16]$. Since the boundaries of the holes handled are usually made up of a B-spline curve, conserving continuity is more important for these holefilling algorithms. Ideally, holefilling algorithm should posses the following properties: (1) able to cover an arbitrary hole for any model (robustness), (2) capable of filling large holes in a reasonable amount of time (efficiency), (3) enable the patched surface to match the missing geometry well (precision). Unfortunately, due to the complexity and diversity of the holes, no existing hole-filling methods satisfy all the above desirable properties. In this thesis, we present a novel hole-filling algorithm for mesh models. The advancing front mesh technique is employed first to generate a new triangular mesh to cover the existing hole. Then we utilize the Poisson equation to optimize the new mesh. The algorithm is intended to be simple, fast and robust. The main advantage of algorithm in this thesis is can preserve geometric features to certain extent. Moreover, the holefilling mesh models are of excellent quality for engineering.

\section{Main Mathmatical Tool}

There is three important mathmatical tools in this thesis, one is least-squares support vector machines, another is lapacian on triangular mesh surface and the last one is discrete possion equation. As mentioned above, keeping geometric features is very important to hole filling algorithm in many applications. Therefore, how to enable the patched surface to match the missing geometry well (precision) is core problem in this thesis.

\subsection{Lapacian on Triangular Mesh Surface}

Lapacian is the most popular mathmatic tool to describe geomatic features on triangular mesh surface. A local film of one vertex on triangular mesh model surface comprised by vertex $V_{i}$ and vertexs on one edge. Fig. 1 is is local film of vertex $V_{i}$. 


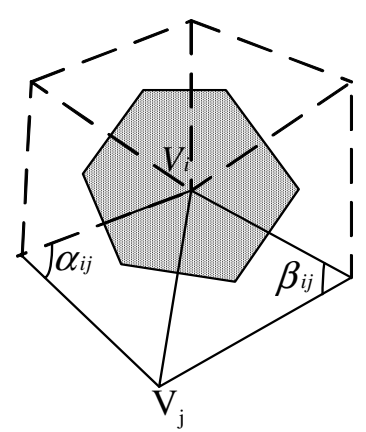

Fig. 1. Local flim of $V_{i}$

Eq (1) is Lapacian of vertex $\mathrm{V}_{\mathrm{i}}, w_{i j}$ is weight coefficient. Eq (2) and Eq (3) is calculating formulas for weight coefficient.

$$
\begin{gathered}
\delta_{i}=\sum_{\mathrm{v} \in \mathrm{N}(\mathrm{i})} w_{i j}\left(v_{j}-v_{i}\right) \\
w_{i j}=\frac{\omega_{i j}}{\sum_{v_{j \in N}(i)} \omega_{i j}} \\
\omega_{i j}=\cot \alpha_{i j}+\cot \beta_{i j}
\end{gathered}
$$

$\delta_{i}$ is cotangent lapacian of $\mathrm{V}_{\mathrm{i}}$ calculating by $\mathrm{Eq}(1), \mathrm{Eq}(2)$ and $\mathrm{Eq}$ (3).

$$
\bar{k}_{i} n_{i}=\delta_{c i}=\frac{1}{4 A\left(v_{i}\right)} \sum_{v j \in N(i)}\left(\cot \alpha_{i j}+\cot \beta_{i j}\right)\left(v_{i}-v_{j}\right)
$$

As Eq (4), cotangent lapacian linear approximate mean curvature in value and approximate principal curvature in direction, therefore cotangent lapacian is perfect mathmatic tools to describe geometrical details of triangular mesh. $A\left(v_{i}\right)$ in Eq (4) is Voronoi graph (Shaded area in Fig1).

$$
\begin{gathered}
L=\left|\begin{array}{ccc}
L_{11} & \cdots & L_{1 n} \\
\vdots & L_{i j} & \vdots \\
L_{n 1} & \cdots & L_{n n}
\end{array}\right| \\
L_{i j}=\left\{\begin{array}{cc}
-1 & i=j \\
w_{i j} & v_{j} \in N(i) \\
0 & \text { otherwise }
\end{array}\right. \\
\Delta_{d}=L V_{d}=\left[\delta_{1 d}, \delta_{2 d}, \delta_{2 d}, \cdots \delta_{n d}\right]^{T}, d \in\{x, y, z\} \\
V_{d}=\left[v_{d 1}, v_{d 2}, v_{d 3}, \cdots, v_{d n}\right]^{T}, d \in\{x, y, z\}
\end{gathered}
$$


For the purpose to calculate lapacian on vertex, lapacian matrix (as Eq (5)) has been introduced in this thesis. As Eq (5), $L$ is lapacian matrix of order $n \times n, n$ is the number of vertexes in computational domain.

Form of $L_{i j}$ in $\mathrm{Eq}$ (5) such as $\mathrm{Eq}(6)$, and $V_{d}$ is component of the vertex coordinates in a coordinate axis, $\Delta d$ is component of the lapacian in acoordinate axis.

\subsection{Least-Squares Support Vector Machines}

least-squares support vector machines is introduced by Suykens first, in this method the least square linear system be used as loss function, thus outstanding advantages of this method is high speed and robustness. The application of least-squares support vector in this thesis is a typical regression problem, regression purpose is optimize fuction $f(x)$. The training sample set in this thesis is components of spatial coordinate around the hole area mesh vertices in the same coordinate axis.

$$
S=\left\{\left(V_{i d}, \delta_{i d}\right), V_{i d} \in R, \delta_{i d} \in R\right\}_{i=1}^{l}
$$

As in $\mathrm{Eq}(9), d \in\{x, y, z\}, l$ is the of samples in training sample set. Based on the structural risk minimization principle, and considering the complexity and the fitting error fuction, the regression problem can be described as the following optimization problem (as Eq (10) and Eq (11)).

$$
\begin{gathered}
\delta_{i d}=w^{T} \varphi\left(V_{i d}\right)+b+e_{i} \\
\min Q(w, b, e)=\frac{1}{2}\|w\|^{2}+\frac{\gamma}{2} \sum_{i=1}^{l} e_{i}^{2}
\end{gathered}
$$

As in Eq (11), $w$ is weighting vector, $e_{i}$ is slack variable, $\gamma$ is regularization parameter, deviation variable, in the purpose to solve above optimizatoin problem, Lagrange fuction need to be established. (As Eq (12))

$$
\begin{gathered}
L(w, b, e, a)=Q(w, b, e)-\sum_{i=1}^{l} a_{i}\left[w^{T} \phi\left(x_{i}\right)+b+e_{i}-y_{i}\right] \\
\left\{\begin{array}{l}
\frac{\partial L}{\partial w}=0 \Rightarrow w-\sum_{i=1}^{l} a_{i} \phi\left(x_{i}\right)=0 \\
\frac{\partial L}{\partial b}=0 \Rightarrow \sum_{i=1}^{l} a_{i}=0 \\
\frac{\partial L}{\partial e_{i}}=0 \Rightarrow C e_{i}-a_{i}=0 \\
\frac{\partial L}{\partial a_{i}}=0 \Rightarrow w^{T} \phi\left(x_{i}\right)+b+e_{i}-y_{i}=0
\end{array}\right.
\end{gathered}
$$

Write $\mathrm{Eq}(13)$ into matrix form, and expunction $w$ and $e$ can get Eq (12). 


$$
\left[\begin{array}{cc}
0 & \bar{I}^{T} \\
\bar{I} & \Omega+\gamma^{-1} I
\end{array}\right]\left[\begin{array}{l}
b \\
a
\end{array}\right]=\left[\begin{array}{l}
0 \\
y
\end{array}\right]
$$

In Eq (14) $\Omega_{i j}=K\left(x_{i}, x_{j}\right)$, and Solve formula (12) to get regression function as Eq (15).

$$
f(x)=\sum_{i=1}^{l} a_{i} K\left(x, x_{i}\right)+b
$$

\subsection{Discrete Possion Equation}

In our implementation, we choose the Poisson equation with Dirichlet boundary conditions to refine the patch mesh. The Poisson equation with the Dirichlet boundary is formulated as $\Delta f=\operatorname{divh}, f|\partial \Omega=f *| \partial \Omega$, where $f$ is unknown scalar fuction $h$ is the guidance vector filed, $\operatorname{div} h$ is the divergence of $h$, and $f *$ is a known scalar function providing the boundary condition. It can be verified that the Poisson equation is the equivalent to the minimization problem as Eq (16).

$$
\min _{f} \int|\nabla f-h|^{2}, \text { with } f|\partial \Omega=f *| \partial \Omega
$$

The discrete Poisson equation is actually a sparse linear system $A x=b$, where the unknown vector $x$ represents special coordinates of all vertices on the reconstructed patch mesh, the coefficient matrix $A$ is determined by Eq (5), and the vector $\boldsymbol{b}$ is a known vector field obtained from the collection of divergence values at all boundary vertices formulated by $\mathrm{Eq}$ (1), which is taken as the boundary condition. The Poisson equation implies that in order to reconstruct the patch mesh we need a guidance vector field defined on the triangles of the patch mesh.

\section{$3 \quad$ Hole-Filling Algorithm}

Algorithm in this thesis can be divided into three following parts. (1) The hole boundary pre-treament: Include a hole boundary indentification and boundary edge pre-trement. (2) Initial patch mesh generation : Include initialize the front using the boundary vertices of the hole; Calculate the angle between two adjacent boundary edges; Starting create new triangles on the plane determined by two adjacent edge until the whole hole has been patched by all newly created triangles. (3) Filling patch refinement based on Poisson equation: Include gather train sample and regression function's solving of least-squares support vector machine; re-positioned vertices of filling patch by solving poisson equation in order to make the patch mesh connect the boundary vertices smoothly and approximate the missing geometry more accurately is employed to refine the patch mesh. 


\subsection{Hole Boundry Pretreament}

There is two steps in this section: hole boundary indentification and boundary edge pretrement. Since a vertex-based topological structure is used in this work, all boundary vertices can be easily identified by checking the numbers of their 1-ring triangles and 1-ring edges, in this thesis octree be used in this to increase indentification speed. The concrete step of boundary indentification as follows:

Step1: Compute center of each triangle patch and contaction betwween the center and its vertices;

Step2: Generate octree based on triangle patch centers;

Step3: Searching octree, and detecting topology connection between its edges, if one edge belong to one triangle patch, the edge is boundary edge;

Step4: Distinguishing different boundary loop by vertex geometric connectivity, showing different holes.

The second step in this section is boundray edge pretrement. Because data information of hole area in not complete, shape of reconstructed triangle patch is not always good, some patch is long and narrow triangle. In propose to reduce influence of bad shape triangle patch, edge pre-treatment must do before filling the mesh hole. The concrete step as follows:

$v_{i}(i=1,2, \cdots, n)$ is $n$ vertices on hole boundary, $\varepsilon$ is edges on hole boundary.

Step1: Compute average length of edges on hole boundary $L_{\text {ave }}$.

Step2: Traversal all edge on hole boundary, if length of one edge $\left(\mathcal{E}_{i j}=\left(v_{i}, v_{j}\right)\right)$ $L_{\varepsilon i j}>k \cdot L_{a v e}$, compute middle point $v_{\text {new }}$ of $\mathcal{E}_{i j}$.

Step3 : Take $v_{\text {new }}$ as new boundary point, and delete $\mathcal{E}_{i j}$ and triangle patch $v v_{i} v_{j}$, add edge $v_{i} \nu_{n e w} 、 v_{i} v_{n e w}$ and triangle patch $v_{i} \nu v_{n e w} 、 v_{n e w} v v_{j}$, as shown in Fig 2.

At last iterate step3, until boundary edge didn't change.
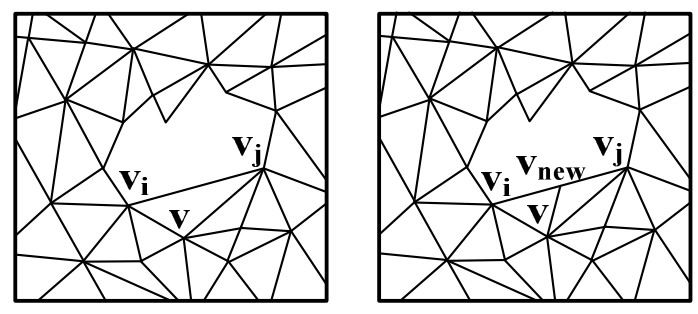

Fig. 2. Hole boundary Pretreatment

\subsection{Initial Patch Mesh Generation}

In this thesis, we adopt the advancing front mesh (AFM) technique to generate an initial patch mesh over the hole. The method consists of the following six steps:

Step 1: Initialize the front using the boundary vertices of the hole.

Step 2: Calculate the angle $\theta_{i}$ between two adjacent boundary edges ( $\boldsymbol{e}_{i}$ and $\boldsymbol{e}_{i+1}$ ) at each vertex $V_{i}$ on the front. 
Step 3 : Starting from the vertex $V_{i}$ with the smallest angle $\theta_{i}$, create new triangles on the plane determined by $\boldsymbol{e}_{i}$ and $\boldsymbol{e}_{i+1}$ with the three rules shown in Fig. 3 .

Step 4 : Compute the distance between each newly created vertex and every related boundary vertex; if the distance between them is less than the given threshold, they are merged.

Step 5 : Update the front.

Step 6 : Repeat Steps 2 through 5 until the whole region has been patched by all newly created triangles.

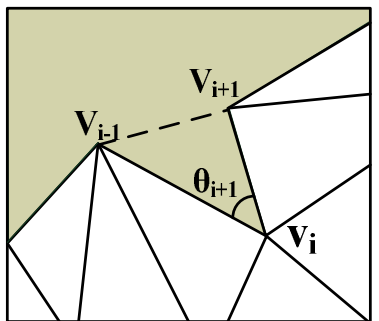

a

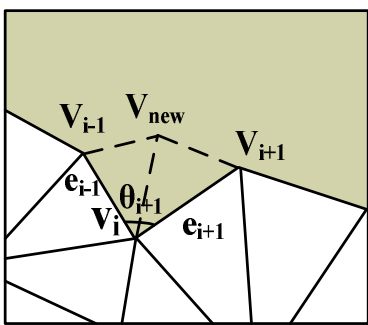

b

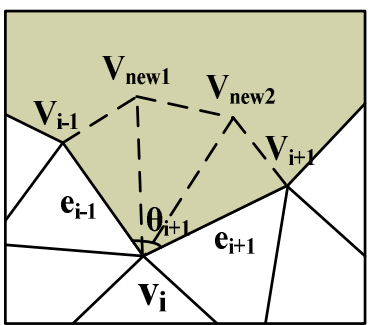

c

Fig. 3. Rules for creating triangles: $\mathrm{A} \theta_{\mathrm{i}} \leq 75^{\circ} ; . \mathrm{b} 75<\theta_{\mathrm{i}} \leq 135^{\circ}$; c $\quad \theta_{\mathrm{i}}>135^{\circ}$

\subsection{Establish the Training Sample Set}

Up to the present all mesh hole filling algorithm deduce geometric characteristic of filling patch depend on geometric characteristic on initial mesh surface surrounding the hole. In this thesis we take lapacian and euclidean coordinate on vertices surrounding the hole boundary as training sample set to least-squares support vector machines.

In case of $V_{e}$ is vertex set on hole boundray $B, S V_{j}(j=0,1, \cdots k)$ is $j$ layer of vertex around hole boundary, in practice there is four step in the traning sample collection method.

Step1 : Take vertex $v_{i}$ on hole boundary, search vertex in same traingle patch with $v_{i}$, if that vertex does not in $V_{e}$, put it in $S V_{1}$.

Step2 : Traversing every vertex in arrays $V_{e}$, repet step1, than gathered vertices on the first layer from hole boundary and put these vertices in $S V_{1}$.

Step3 : To vertices in $S V_{j}(j=1,2 \cdots k-1)$ search vertex in same traingle patch with them, than put vertices not in $S V_{j}$ and $S V_{j-1}$ into $S V_{j+1}$.

Step4 : Iterate step3, until the setted up layer number of $S V_{j}(j=0,1, \cdots k)$ attained. Under this circumstances all vertices and its lapacian consist training samples set, the set of sample points as Fig 4. 


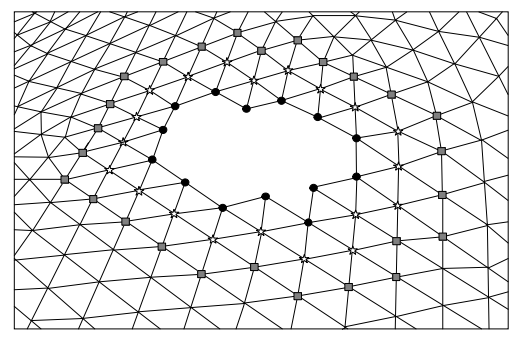

Fig. 4. Training sample set for least-squares support vector machines

\subsection{Filling Patch Refinement Based on Poisson Equation}

According to method mentioned in section 2.2, Put training sample set in leastsquares support vector machines, get three regression fuction : $f_{x}(x), f_{y}(x), f_{z}(x)$. By three regression fuction we can get new lapacian field that the poisson equation requires. Once a discrete lapacian fields is given, we consider the new lapacian field as the lapacian field in the Poisson equation, a piecewise continuous scalar function, a connected mesh, can be reconstructed, which computes euclidean coordinate of vertices on filling patch in the least squares sense.

In this thesis, process of computing new coordinates of vertices on filling patch is solution of $n \times n$ linear equstions as (18).

$$
\begin{gathered}
V_{d}^{\prime}=\left[v_{d 1}^{\prime}, v_{d 2}^{\prime}, v_{d 3}^{\prime}, \cdots, v_{d n}^{\prime}\right]^{T}, d \in\{x, y, z\} \\
L_{V n} V^{\prime}{ }_{n d}=\Delta^{\prime}{ }_{d n} \\
\Delta_{d n}^{\prime}=L_{V n} V_{d}=\left[f_{d}\left(v^{\prime}{ }_{1} d\right), f_{d}\left(v^{\prime}{ }_{2} d\right), \cdots f_{d}\left(v^{\prime}{ }_{n} d\right)\right]^{T}, d \in\{x, y, z\}
\end{gathered}
$$

$L_{V n}$ in (18) is contangent lapacian matrix established by method mentioned in section 2.1 of filling patch, $\Delta_{d n}^{\prime}$ in (19) is one component of lapacian of vertices on reconstructed filling patch. $f_{d}\left(v_{n}^{\prime} d\right)$ in (19) is one regression fuction.

\section{$4 \quad$ Implementation and Analysis}

The proposed hole-filling algorithm has been implemented with VisualC++2010 and OpenGL. All experimental results in this paper were obtained on a $20 \mathrm{GHz}$ Pentium IV personal computer with 1024MB memory. Many examples have been used to test the robustness, efficiency and accuracy of the method.

Figure 5a shows head model, there are a large number of geometric details on this mesh model. Figure $5 \mathrm{~b}$ shows head model with hole in the part of hair. In this section the hole-filling result on head model by direct filling algorithm, algorithm in reference[17] and algorithm in this thesis. The reason of taking algorithm in 
reference[17] compare to algorithm in this thesis is the algorithm in reference[17] is based on solution of possion equation same to algorithm in this thesis, the differene between them is least-squares support vector machine has been used in algorithm in this thesis to estimate geometric details of filling patch, so the comparison of two algorithms show the advantage of algorithm in this thesis at keeping geomatric style between filling patch and other part of mesh model.

Figure 6 depicts filling result by direct filling algorithm, obviously very routh. Figure 7 depicts filling result by filing algorithm in reference[17], as shown in figure 7, filling result is very good, mesh quality of filling patch is well two, the only disfigurement is filling patch and other part of model is not coincident in geomatric style at all. Figure 8 depicts filling result by algorithm in this thesis, from the picture we can saw that mesh quality is as good as in Figure 7, and keeping geomatric style between filling patch and and other part of mesh very well.

Table 1 is error evaluaton for algorithm in reference[17] and in thesis. Table 2 is ranning time of two algorithms states above.

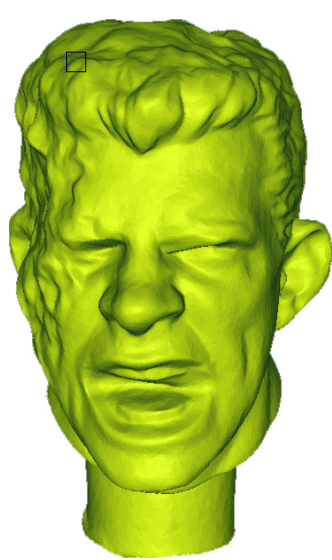

a

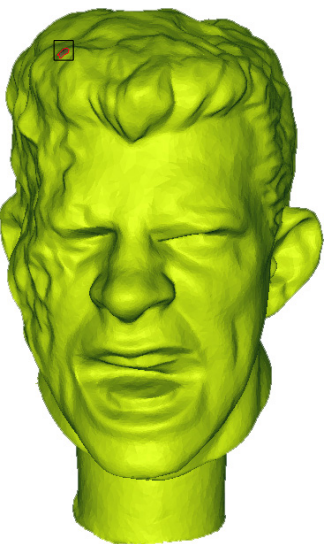

b

Fig. 5. Preliminary head model and head model with hole

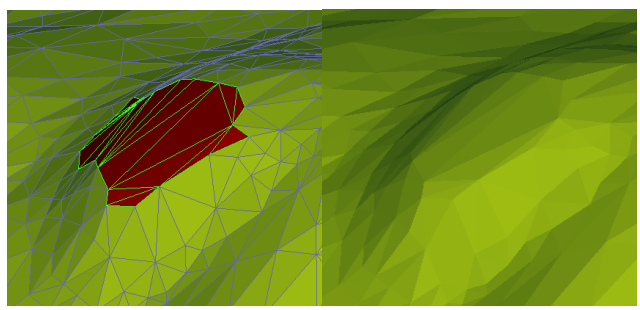

Fig. 6. Filling result by direct filling algorithm 


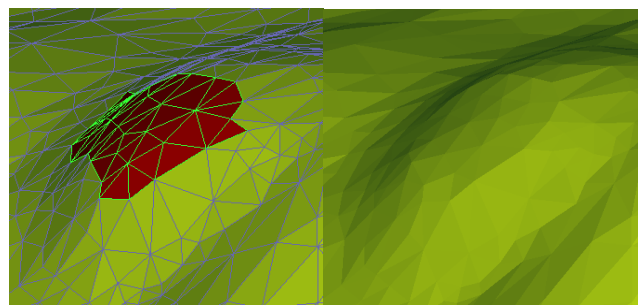

Fig. 7. Filling result by filling alorithm in reference 17

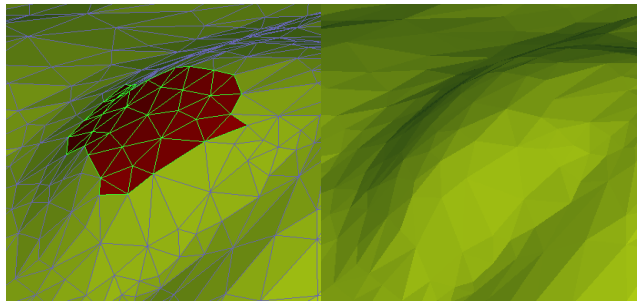

Fig. 8. Filling result by filling alorithm in this thesis
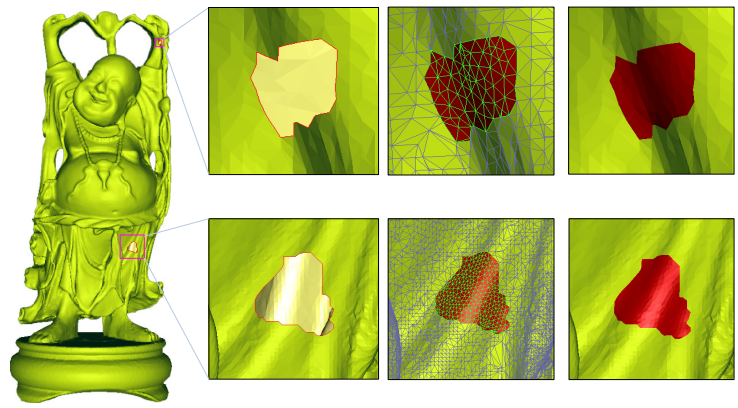

Fig. 9. Filling result on happyvrip model by filling alorithm in this thesis

Table 1. Error evaluation

\begin{tabular}{ccccc}
\hline Algorithm & $\begin{array}{c}\text { Vertices num } \\
\text { on filling patch }\end{array}$ & $\begin{array}{c}\text { Triangle num } \\
\text { on filling patch }\end{array}$ & Averange distance & Averange error \\
In reference 17 & 28 & 69 & $0.05(\mathrm{~mm})$ & 0.0015 \\
In this thesis & 31 & 75 & $0.042(\mathrm{~mm})$ & 0.00142 \\
\hline
\end{tabular}

Table 2. Computing time

\begin{tabular}{ccccc}
\hline Algorithm & $\begin{array}{c}\text { Vertices num } \\
\text { on filling patch }\end{array}$ & $\begin{array}{c}\text { Triangle num } \\
\text { on filling patch }\end{array}$ & $\begin{array}{c}\text { Time of Initial patch } \\
\text { mesh generation }\end{array}$ & $\begin{array}{c}\text { Time of solving } \\
\text { possion equation }\end{array}$ \\
In reference 17 & 28 & 69 & $10.6 \mathrm{~ms}$ & $15.7 \mathrm{~ms}$ \\
In this thesis & 31 & 75 & $10.4 \mathrm{~ms}$ & $15.9 \mathrm{~ms}$ \\
\hline
\end{tabular}

In this thesis the distances between the vertices on the patch mesh and the original analytic surface are used to evaluate the precision of our algorithm. The quotient of the average distance and the square root of analytic surface area is considered as the 
error of our algorithm. From table 1 and table 2 we can see that by algorithm in this thesis we can gen higher precision while spend similar time.

Fig 9 depicts filling result by algorithm in this thesis on big hole, from the picture we can see that the algorithm work very well in keeping geomatric style between filling patch and other part when the hole need to fill is compared bigger.

\section{Conclusion}

The innovation point of this thesis is taking lapacian of vertices on filling patch as output of regression fuction and reconstruction filling patch by solving possion equation. The advantage of this algorithm is use lapacian to depicte geomatric details of mesh surface model, thereforce makeing filling mesh patch and original mesh surface inosculated more naturally.

Limitation of algorithm in this thesis is filling result may lose some geomatric details when the hole is too big, this limitation is existing in all popular mesh surface filling algorithms. The method dealing with this problem is taking whole geometric characteristic of mesh modle as reference while filling the mesh surface hole, this is also research area of this thesis.

\section{References}

1. Davis, J., Marschner, S.R., Garr, M., Levoy, M.: Filling holes in complex surfaces using volumetric diffusion. In: First International Symposium on 3D Data Processing Visualization and Transmission, Padova, pp. 428-438 (2002)

2. Guo, T.-Q., Li, J.-J., Weng, J.-G., Zhuang, Y.-T.: Filling holes in complex surfaces using oriented voxel diffusion. In: 2006 International Conference on Machine Learning and Cybernetics, pp. 4370-4375. IEEE, Los Alamitos (2006)

3. Ju, T.: Robust repair of polygonal models. In: Processing of SIGGRAPH, Los Angeles, CA, USA, August 8-12, pp. 888-895. ACM Press, New York (2004)

4. Joshua, P., Szymon, R.: Atomic volumes for mesh completion. In: Proceedings Eurographics Symposium on Geometry Processing, Dublin, Ireland, August 29-September 2, pp. 33-41. Blackwell Press (2005)

5. Brunton, A., Wuhrer, S., Shu, C., Bose, P., Demaine, E.D.: Filling holes in triangular meshes by curve unfolding. In: IEEE International Conference on Shape Modeling and Applications, pp. 66-72 (2009)

6. Li, B.J., Zhang, X.K., Zhou, P., Hu, P.: Mesh parameterization based on one-step inverse forming. Comput. Aided Des. 42(7), 633-640 (2010)

7. Gao, S., Zhao, W., Lin, H., Yang, F., Chen, X.: Feature suppression based cad mesh model simplification. Computer-Aided Design 42(12), 1178-1188 (2010)

8. Carr, J., Beatson, R., Cherrie, J., Mitchell, T., Fright, W., McCallum, B.: Reconstruction and representation of 3D objects with radial basis functions. In: Processing of SIGGRAPH, Los Angeles, CA, August 12-17, pp. 67-76. ACM Press, New York (2001)

9. Liepa, P.: Filling hole in meshes. In: Proceedings Eurographics Symposium on Geometric Processing, Granada, Spain, September 1-6, pp. 200-207. Blackwell Press (2003)

10. Jun, Y.: A piecewise hole-filling algorithm in reverse engineering. Comput. Aided Des. 22(8), 263-270 (2005) 
11. Chen, C.Y., Cheng, K.Y., Liao, H.Y.M.: A sharpness dependent approach to 3D polygon mesh hole filling. In: Proceedings of Eurographic, Trinity College, Dublin, Ireland, August 29-September 2, pp. 13-16. Blackwell Press (2005)

12. Chen, C.Y., Cheng, K.Y.: A sharpness dependent filter for mesh smoothing. Comput. Aided Geom. Des. 22(5), 376-391 (2005)

13. Chen, C.Y., Cheng, K.Y., Liao, H.Y.M.: Fairing of polygon meshes via Bayesian discriminate analysis. In: The 12th International Conference in Central Europe on Computer Graphics, Visualization and Computer Vision, Plzen-Bory, Czech Republic, pp. 175-182. UNION Agency-Science Press (2004)

14. Lou, R., Pernot, J.-P., Mikchevitch, A., Véron, P.: Merging enriched finite element triangle meshes for fast prototyping of alternate solutions in the context of industrial maintenance. Comput. Aided Des. 42(8), 670-681 (2010)

15. Ju, T.: Fixing geometric errors on polygonal models: a survey. Journal of Computer Science and Technology 24(1), 19-29 (2009)

16. Li, Z., Meek, D.S., Walton, D.J.: Polynomial blending in a mesh hole-filling application. Computer-Aided Design 42, 340-349 (2010)

17. Zhao, W., Gao, S.M., Lin, H.W.: A robust hole-filling algorithm for triangular mesh. The Visual Computer: International Journal of Computer Graphics 23(12), 987-997 (2007) 